\title{
Randomized trial of mediastinal lymph node sampling versus complete lymphadenectomy during pulmonary resection in the patient with NO or N1 (less than hilar) non-small cell carcinoma: Results of the American College of Surgery Oncology Group Z0030 Trial
}

Gail E. Darling, MD, ${ }^{\mathrm{a}}$ Mark S. Allen, MD, ${ }^{\mathrm{b}}$ Paul A. Decker, MS, ${ }^{\mathrm{b}}$ Karla Ballman, PhD, ${ }^{\mathrm{b}}$ Richard A. Malthaner, MD, ${ }^{\mathrm{c}}$ Richard I. Inculet, MD, ${ }^{\mathrm{c}}$ David R. Jones, MD, ${ }^{\mathrm{d}}$ Robert J. McKenna, MD, Rodney J. Landreneau, MD, ${ }^{\mathrm{f}}$ Valerie W. Rusch, MD, ${ }^{\mathrm{g}}$ and Joe B. Putnam, Jr, MD

Objective: To determine whether mediastinal lymph node dissection improves survival compared with mediastinal lymph node sampling in patients undergoing resection for $\mathrm{N} 0$ or nonhilar N1, T1, or T2 non-small cell lung cancer.

Methods: Patients with non-small cell lung cancer underwent sampling of 2R, 4R, 7, and 10R for right-sided tumors and 5, 6, 7, and 10L for left-sided tumors. If all tumors were negative for malignancy, patients were randomized to no further lymph node sampling (mediastinal lymph node sampling) or complete mediastinal lymph node dissection.

Results: Of 1111 patients randomized, 1023 (mediastinal lymph node sampling in 498, mediastinal lymph node dissection in 525) were eligible and evaluable. There were no significant differences between the 2 groups in terms of demographics, Eastern Cooperative Oncology Group status, histology, cancer location, type or extent of resection, and pathologic stage. Occult $\mathrm{N} 2$ disease was found in 21 patients in the mediastinal lymph node dissection group. At a median follow-up of 6.5 years, 435 patients $(43 \%)$ have died: mediastinal lymph node sampling in 217 $(44 \%)$ and mediastinal lymph node dissection in $218(42 \%)$. The median survival is 8.1 years for mediastinal lymph node sampling and 8.5 years for mediastinal lymph node dissection $(P=.25)$. The 5 -year disease-free survival was $69 \%$ (95\% confidence interval, 64-74) in the mediastinal lymph node sampling group and $68 \%(95 \%$ confidence interval, 64-73) years in the mediastinal lymph node dissection group $(P=.92)$. There was no difference in local $(P=.52)$, regional $(P=.10)$, or distant $(P=.76)$ recurrence between the 2 groups.

Conclusions: If systematic and thorough presection sampling of the mediastinal and hilar lymph nodes is negative, mediastinal lymph node dissection does not improve survival in patients with early stage non-small cell lung cancer, but these results are not generalizable to patients staged radiographically or those with higher stage tumors. (J Thorac Cardiovasc Surg 2011;141:662-70)

\section{Earn CME credits at}

http://cme.ctsnetjournals.org

From the University of Toronto, ${ }^{\mathrm{a}}$ Toronto, Ontario, Canada; Mayo Clinic, ${ }^{\mathrm{b}}$ Rochester, Minn; London Health Sciences Center, ${ }^{\mathrm{c}}$ London, Ontario, Canada; University of Virginia, ${ }^{\mathrm{d}}$ Charlottesville, Va; Cedars Sinai Medical Center, ${ }^{\mathrm{e}}$ Los Angeles, Calif; University of Pittsburgh, ${ }^{\mathrm{f}}$ Pittsburgh, Pa; Memorial Sloan Kettering Cancer Center, ${ }^{\mathrm{g}}$ New York, NY; and Vanderbilt University Medical Center, ${ }^{\mathrm{h}}$ Nashville, Tenn.

This study was coordinated by the American College of Surgeons Oncology Group and is supported by funding from the US National Cancer Institute to the American College of Surgeons Oncology Group, Grant U10 CA 76001.

Disclosures: Authors have nothing to disclose with regard to commercial support.

Read at the 90th Annual Meeting of The American Association for Thoracic Surgery, Toronto, Ontario, Canada, May 1-5, 2010.

Received for publication April 30, 2010; revisions received Oct 18, 2010; accepted for publication Nov 2, 2010.

Address for reprints: Gail E. Darling, MD, University Health Network, Toronto General Hospital 9N-955, 200 Elizabeth St, Toronto, Ontario, Canada M5G 2C4 (E-mail: gail.darling@uhn.on.ca).

$0022-5223 / \$ 36.00$

Copyright ( 2011 by The American Association for Thoracic Surgery doi:10.1016/j.jtcvs.2010.11.008
Lung cancer is the leading cause of cancer deaths. ${ }^{1}$ Nonsmall cell lung cancer (NSCLC) may be curable by surgical resection; however, even tumors that appear localized by imaging may have lymph node metastases. Lymph node assessment is important for accurate staging of NSCLC. However, the extent of lymph node removal required and the impact of mediastinal node removal on survival are controversial. Unfortunately, in a pattern of care study, only $57.3 \%$ of patients had any mediastinal nodes removed at the time of pulmonary resection. ${ }^{2}$

Studies addressing the survival benefit of mediastinal lymph node dissection (MLND) have been inconclusive, with only 1 of 3 previous randomized trials reporting a survival advantage. ${ }^{3}$ Proponents argue that MLND, by removing occult N2 disease, would decrease recurrence and increase survival. However, distance metastases as the first site of recurrence develop in two thirds of patients with $\mathrm{N} 2$ disease. ${ }^{4}$

The American College of Surgery Oncology Group (ACOSOG) Z0030 study was a randomized, multi-institutional, 


\section{Abbreviations and Acronyms \\ ACOSOG $=$ American College of Surgery \\ Oncology Group \\ $\mathrm{CI}=$ confidence interval \\ CT = computed tomography \\ MLND = mediastinal lymph node dissection \\ MLNS = mediastinal lymph node sampling \\ NSCLC $=$ non-small cell lung cancer \\ VATS $=$ video-assisted thoracic surgery}

prospective trial of MLND versus mediastinal lymph node sampling (MLNS) during pulmonary resection for patients with early-stage NSCLC. This study addresses the question of whether survival is improved by MLND compared with MLNS in early-stage NSCLC and compares recurrence patterns.

\section{MATERIALS AND METHODS}

The protocol was approved by a central institutional review board and the institutional review board at each participating institution. All patients provided written informed consent before trial enrollment.

Participating surgeons were required to read a detailed description of the technique of MLND and watch an instructional video. All operative notes and pathology reports were reviewed by the principal investigators (M.S.A. or G.E.D.) for completeness of the mediastinal dissection. Specifically, it was ascertained that additional lymph nodes were removed during MLND per protocol. Lymph nodes were named according to the American Thoracic Society lymph node stations. ${ }^{5}$ Eligibility requirements and methods have been published. ${ }^{6}$ Patients with proven NSCLC underwent a rigorous mediastinal and hilar lymph node sampling per protocol before randomization. For tumors in the right lung, lymph node stations $2 \mathrm{R}, 4 \mathrm{R}, 7$, and 10R were sampled. For tumors in the left lung, stations 5, 6, 7, and 10L were sampled. Any suspicious lymph nodes were also biopsied. The surgeon had the option of sampling by mediastinoscopy $(2 \mathrm{R} / \mathrm{L}, 4 \mathrm{R} / \mathrm{L}$, and 7), thoracotomy, or video-assisted thoracic surgery (VATS). Station 10 nodes were sampled at thoracotomy or VATS. If all sampled lymph nodes showed no evidence of cancer on frozen-section examination, patients were randomized intraoperatively via telephone by the central coordinating center to lymph node sampling only (MLNS) with no further lymph node removal or to complete MLND.

MLND was performed according to a previously described protocol. ${ }^{7}$ For tumors on the right side, all lymph tissue was removed from an area bounded by the takeoff of the right upper lobe bronchus, innominate artery, superior vena cava, and trachea (stations $2 \mathrm{R}$ and $4 \mathrm{R}$ ). Lymph nodes in the prevascular area, adjacent to the superior vena cava, and retrotracheal nodes were removed. Complete MLND for tumors on the left involved removing all lymph tissue between the phrenic and vagus nerves extending down to the left main stem bronchus (stations 5 and 6). At the completion of the dissection, the aortopulmonary window was free of lymph tissue and the recurrent nerve was preserved. Regardless of the side of the tumor, complete subcarinal lymph node dissection was performed, removing all lymph tissue caudal to the carina and both left and right mainstem bronchi (station 7). Lymph nodes in the inferior pulmonary ligament and adjacent to the caudal half of the esophagus were also removed (stations 8 and 9). When the dissection was complete, the mainstem bronchi, posterior pericardium, and esophagus were free of all lymph tissue. In both arms, all lobar and interlobar lymph nodes were resected during the lung resection.

\section{Statistical Methods}

The target accrual for this study was 1037, with the final analysis to occur after 459 deaths. This was determined on the basis of the assumption that after surgery there would be 4 pathologic stage groups-pT1N0, pT2N0, pN1, and pN2/pIIIA, with expected proportions of $40 \%, 30 \%$, $15 \%$, and $15 \%$, respectively. For calculating the sample size, it was assumed that the 5-year survival in the sampling arm for the 4 groups would be $75 \%, 60 \%, 40 \%$, and $30 \%$, respectively. However, observed proportions of the 4 pathologic stage groups in the sampling arm of the study-pT1N0, pT2N0, pN1, and pN2/pIIIA- were $41 \%, 41 \%, 13 \%$, and $4 \%$, respectively. In addition, the observed 5-year survival in the sampling arm for the 4 groups was $74 \%, 59 \%, 44 \%$, and $27 \%$, respectively. This translated to a weighted yearly death hazard rate of 0.0998 (assuming survival time is exponentially distributed). An $8 \%$ higher 5-year survival in arm 2 was considered clinically important. Under exponential distribution assumptions, this translated to a death hazard rate that is $25 \%$ less, or a hazard ratio of $0.75(0.0751 / 0.0998)$. In calculating the sample size for survival, the following specifications were made: 1 -sided significance level of .05, statistical power of 0.90 , uniform patient accrual, a 5-year accrual period, and a follow-up period of 5 years.

Patient and surgical characteristics were compared between treatment arms using the chi-square test for categoric variables, Kruskal-Wallis test for continuous variables, and Wilcoxon test for ordinal variables. $\mathrm{Cu}$ mulative time to event (survival, recurrence) probabilities was estimated using the Kaplan-Meier method. The log-rank test was used to compare survival and recurrence by treatment group. For the recurrence analysis, deaths were censored. An additional comparison of local, regional, and distant recurrence was performed using a cumulative incidence approach. This methodology was used to account for competing risks when determining recurrence rates because only first sites of recurrence were recorded. Death was considered a competing event. One-sided statistical tests were used for the primary end point of overall survival.

\section{RESULTS}

From June 1999 to February 2004, 1111 patients were randomized by 102 different surgeons from 63 institutions. All participating surgeons were general thoracic surgeons and diplomats of the American Board of Thoracic Surgery or equivalent. After randomization, retrospective review found 155 patients $(13.9 \%)$ to be ineligible (Figure 1). In 67 of these patients, the reason for ineligibility was minor (eg, timing violation), and these patients were included in this analysis. The remaining 88 patients (57 in the MLNS group and 31 in the MLND group) were excluded for major violations, including incorrect clinical stage in 29 patients, inadequate lymph node sampling in 14 patients, benign disease in 6 patients, insufficient documentation in 5 patients, and other reasons in 34 patients. All analyses were performed on the 1023 (MLNS in 498 and MLND in 525) eligible subjects, and additional intent-to-treat analyses were performed on all randomized subjects. Details of the demographic profile have been published (Table 1). ${ }^{6}$ The median age was 68 years (range, 23-89 years), with a slight male predominance $(\mathrm{n}=529 ; 52 \%) ; 955(93 \%)$ were white, $46(5 \%)$ were black, and $22(2 \%)$ were of other racial backgrounds. Most patients had an Eastern Cooperative Oncology Group performance score of zero $(n=688 ; 67 \%)$ or $1(n=307 ; 30 \%)$. There were no clinically significant differences in demographic profile or 


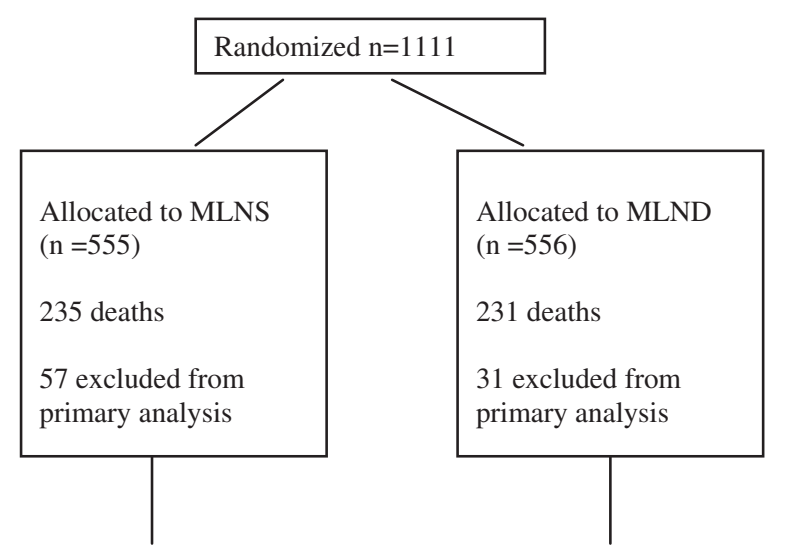

\begin{tabular}{|l|l|}
\hline MLNS & MLND \\
Analyzed* $(\mathrm{n}=498)$ & Analyzed* $(\mathrm{n}=525)_{217 \text { deaths }}^{218 \text { deaths }}$ \\
\hline
\end{tabular}

* Note: intent to treat analyses were also performed.

FIGURE 1. Consort diagram. MLNS, Mediastinal lymph node sampling; $M L N D$, mediastinal lymph node dissection.

Eastern Cooperative Oncology Group status between the study arms.

Approximately $61 \%$ of tumors were located in the upper lobes, and adenocarcinoma was the predominant histology $(42 \%)$ (Table 1). Mediastinoscopy was performed in 303 patients overall; MLNS in 162 patients (33\%) and MLND in 141 patients $(27 \%)(P=.05)$. Per protocol, anatomic pulmonary resections were performed in all patients: lobectomy in 764 patients $(75 \%)$, segmentectomy in 70 patients (7\%), pneumonectomy in 42 patients $(4 \%)$, and bilobectomy in 43 patients $(4 \%)$. Combinations were performed in 100 patients $(10 \%)$. Resection was complete (R0) in 1000 patients $(98 \%)$, incomplete (R1) in 19 patients $(2 \%)$, and grossly incomplete (R2) in 2 patients $(0.2 \%)$. There was no statistical difference in type of resection, operative approach (thoracotomy vs VATS), or completeness of resection between the MLND and MLNS groups ${ }^{6}$ (Table 1).

Only 67 patients $(7 \%)$ underwent resection via VATS. There was no difference in the number of lymph nodes removed by VATS compared with open resections (median nodes removed: 15 vs $19, P=.17$ ). However, more nodes were harvested via lobectomy (median $=18$ ) compared with segmentectomy (median $=14)(P=.006)$.

After prerandomization sampling, a median of 18 additional nodes were removed in those patients randomized to MLND (range, 1-72 for right-sided tumors; 4-69 for leftsided tumors), and 516 patients ( $99 \%$ ) had at least 6 nodes removed from 3 mediastinal node stations. ${ }^{7}$ With MLND, at least 1 additional mediastinal lymph node was removed from each station, with a median of 1 to 4 nodes removed.
TABLE 1. Patient and surgical characteristics of the patients in each arm of the American College of Surgery Oncology Group Z0030 Study

\begin{tabular}{|c|c|c|c|}
\hline & $\begin{array}{c}\text { Lymph node } \\
\text { sampling arm } \\
\quad(\mathrm{n}=\mathbf{4 9 8})\end{array}$ & $\begin{array}{c}\text { Lymph node } \\
\text { dissection arm } \\
(\mathbf{n}=\mathbf{5 2 5}) \\
\end{array}$ & $P^{*}$ \\
\hline Male & $257(52 \%)$ & $272(52 \%)$ & .95 \\
\hline Median age, y (range) & $68(23-89)$ & $67(37-87)$ & .026 \\
\hline \multicolumn{4}{|c|}{ ECOG performance score } \\
\hline 0 & $344(69 \%)$ & $344(66 \%)$ & .34 \\
\hline 1 & $139(28 \%)$ & $168(32 \%)$ & \\
\hline 2 & $15(3 \%)$ & $13(3 \%)$ & \\
\hline \multicolumn{4}{|l|}{ Tumor location $\dagger$} \\
\hline RUL & $193(39 \%)$ & $194(37 \%)$ & .55 \\
\hline RML & $36(7 \%)$ & $29(6 \%)$ & .26 \\
\hline RLL & $88(18 \%)$ & $101(19 \%)$ & .52 \\
\hline LUL & $129(26 \%)$ & $144(27 \%)$ & .58 \\
\hline LLL & $58(12 \%)$ & $64(12 \%)$ & .79 \\
\hline Histology & & & .53 \\
\hline Squamous cell & $132(27 \%)$ & $141(27 \%)$ & \\
\hline Adenocarcinoma & $195(39 \%)$ & $228(44 \%)$ & \\
\hline Large cell & $27(5 \%)$ & $22(4 \%)$ & \\
\hline Bronchoalveolar & $36(7 \%)$ & $32(6 \%)$ & \\
\hline Other NSCLC & $106(21 \%)$ & $99(19 \%)$ & \\
\hline Type of resection $\ddagger$ & & & .66 \\
\hline Segmentectomy & $36(7 \%)$ & $34(7 \%)$ & \\
\hline Lobectomy & $379(76 \%)$ & $385(74 \%)$ & \\
\hline Bilobectomy & $18(4 \%)$ & $25(5 \%)$ & \\
\hline Pneumonectomy & $18(4 \%)$ & $24(5 \%)$ & \\
\hline Combination & $45(9 \%)$ & $55(11 \%)$ & \\
\hline Extent of resection & & & .36 \\
\hline R0 & $488(98 \%)$ & $512(98 \%)$ & \\
\hline $\mathrm{R} 1$ & $10(2 \%)$ & $9(2 \%)$ & \\
\hline R2 & $0(0 \%)$ & $2(0.4 \%)$ & \\
\hline Stage & & & .34 \\
\hline IA & $211(43 \%)$ & $212(41 \%)$ & \\
\hline IB & $205(41 \%)$ & $213(41 \%)$ & \\
\hline IIA & $13(3 \%)$ & $24(5 \%)$ & \\
\hline IIB & $56(11 \%)$ & $41(8 \%)$ & \\
\hline IIIA & $4(1 \%)$ & $22(4 \%)$ & \\
\hline IIIB & $8(2 \%)$ & $11(2 \%)$ & \\
\hline
\end{tabular}

ECOG, Eastern Cooperative Oncology Group; NSCLC, non-small cell lung cancer; $R U L$, right upper lobe; $R M L$, right middle lobe; $R L L$, right lower lobe; $L U L$, left upper lobe; $L L L$, left lower lobe. *Chi-square test, Kruskal-Wallis test, or Wilcoxon test as appropriate. $\dagger$ Counts do not sum to $100 \%$ because some patients had disease that involved multiple lobes. $\ddagger$ The type of resection could not be determined in 4 patients.

The pathologic stages are shown in Table 1. Forty-five patients $(4.4 \%)$ had pathologic stage IIIA or IIIB: 12 in the MLNS arm and 33 in the MLND arm. Positive mediastinal lymph nodes (N2) were discovered in 21 patients who had negative sampling and were randomized to MLND (4\%; 95\% confidence interval $[\mathrm{CI}], 2.5-6.1)$. There were 303 patients who underwent mediastinoscopy for lymph node sampling before randomization. Despite a negative mediastinoscopy, after MLND, 8 patients $(2.6 \%)$ were found to be N2 on final pathology, whereas of the 718 patients who had lymph node sampling at thoracotomy or 


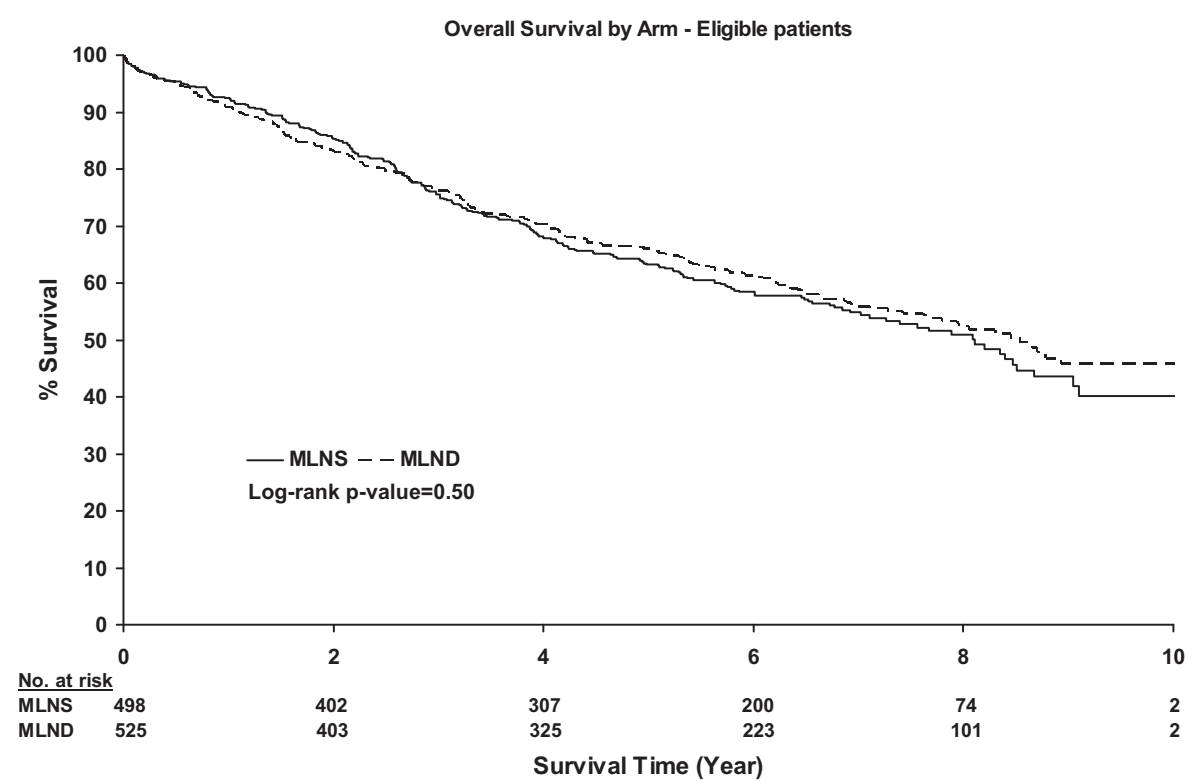

FIGURE 2. Overall survival. MLNS, Mediastinal lymph node sampling; $M L N D$, mediastinal lymph node dissection.

VATS before randomization, $13(1.8 \%)$ were $\mathrm{N} 2$ on final pathology. The reason for designation of stage IIIA/B in the 12 patients in the MLNS arm and 12 patients (nonN2) in the MLND arm included another cancer in the same lobe, tumor less than $2 \mathrm{~cm}$ from carina, involvement of phrenic nerve, and tracheal involvement.

In the group who had initial sampling by mediastinoscopy, 56 patients $(19 \%)$ were found to be N1 positive on final pathology compared with 71 patients $(10 \%)$ who had sampling by thoracotomy or VATS. There was no difference in survival between patients who had their initial nodal assessment by mediastinoscopy and those assessed by thoracotomy or VATS.

At a median follow-up of 6.5 years (range, $0-10.1$ years), $466(42 \%)$ of the 1111 patients have died: $235(42 \%)$ in the MLNS arm and $231(42 \%)$ in the MLND arm. Of the 1023 eligible patients, 217 (44\%) died in the MLNS arm and 218 $(42 \%)$ died in the MLND arm. The median survival was 8.1 years (95\% CI, 7.0-9.0) in the MLNS group and 8.5 years (95\% CI, 7.4-not achieved) in the MLND group $(P=.25$; Figure 2, Table 2). Similar results were observed when the analysis was performed on all 1111 randomized subjects; the median survival in the MLNS group was 8.1 years (95\% CI, 7.0-9.0) versus 8.4 years $(95 \%$ CI, 7.3-not achieved $)$ in the MLND group $(P=.34)$. There were 285 recurrences reported, including 54 local, 74 regional, and 225 distant recurrences in the eligible subjects. Of these, 138 (24 local, 43 regional, and 111 distant) recurrences were in the MLNS arm and 148 (30 local, 31 regional, and 114 distant) recurrences were in the MLND arm. The 5 -year disease-free survival was $69 \%$ (95\% CI, 64-74) in the MLNS arm and 68\% (95\% CI, 64-73) in the MLND arm $(P=.92$; Table 2$)$. Similar results were obtained on all randomized subjects; 5-year disease-free survival was $68 \%(95 \%$ CI, 64-73) in the MLNS group and $67 \%$ (95\% CI, 62-71) in the MLND group $(P=.89)$. An additional analysis was performed separately for T1 $(P=.83)$ and T2 $(P=.63)$ tumors and no differences were observed in disease-free survival between treatment arms (Table 3 ). There was no difference among local $(P=.52)$, regional $(P=.10)$, or distant $(P=.76)$ recurrence between the 2 treatment arms. Similar results were observed for all randomized subjects $(P=.54, P=.24$, and $P=.77$ for local, regional, and distant recurrence, respectively).

\section{DISCUSSION}

This prospective randomized controlled clinical trial found no difference in long-term survival between MLND and MLNS during pulmonary resection for patients with $\mathrm{T} 1$ or T2, N0 or nonhilar N1 NSCLC. We also did not

TABLE 2. Overall and disease-free survival estimates on all eligible patients

\begin{tabular}{ccc}
\hline \multicolumn{2}{c}{$\begin{array}{c}\text { MLNS }(\mathbf{N}=\mathbf{4 9 8}) \text { estimate } \\
\text { Time }\end{array}$} & $\begin{array}{c}\text { MNLD }(\mathbf{n}=\mathbf{5 2 5}) \text { estimate } \\
(\mathbf{9 5} \% \mathbf{C I})\end{array}$ \\
\hline \multicolumn{2}{c}{ Overall survival $\mathbf{C I})$} \\
2 y & $85.1(82.0-88.3)$ & $83.0(79.8-86.4)$ \\
4 y & $67.8(63.7-72.2)$ & $70.5(66.5-74.6)$ \\
6 y & $58.1(53.7-62.9)$ & $61.4(57.1-66.0)$ \\
8 y & $50.9(45.9-56.5)$ & $52.4(47.6-57.7)$ \\
Disease-free survival & \\
2 y & $79.5(75.7-83.4)$ & $80.5(76.9-84.3)$ \\
4 y & $70.6(66.2-75.2)$ & $71.7(67.4-76.2)$ \\
6 y & $65.2(60.4-70.4)$ & $66.3(61.8-71.3)$ \\
8 y & $61.1(55.4-67.3)$ & $59.4(53.8-65.6)$ \\
\hline
\end{tabular}

MLNS, Mediastinal lymph node sampling; $M L N D$, mediastinal lymph node dissection; $C I$, confidence interval. 
TABLE 3. Disease-free survival in T1/T2 eligible patients

\begin{tabular}{lcccc}
\hline & \multicolumn{4}{c}{ Disease-free survival in T1/T2 eligible patients } \\
\cline { 2 - 5 } Time & T1 MLNS estimate (95\% & T2 MLNS estimate (95\% & T1 MNLD estimate (95\% & T2 MNLD estimate (95\% \\
CI) (\%) & CI) (\%) \\
\hline 2 y & CI) (\%) & $75.1(69.5-81.2)$ & $91.0(87.1-95.1)$ & $71.7(66.0-77.9)$ \\
4 y & $79.4(82.5-92.1)$ & $64.7(58.3-71.7)$ & $82.1(76.6-87.9)$ & $62.9(56.7-69.8)$ \\
6 y & $75.0(68.5-82.1)$ & $58.1(51.2-65.8)$ & $73.7(67.1-81.0)$ & $59.0(52.6-66.3)$ \\
8 y & $69.7(61.5-79.0)$ & $54.8(47.0-63.7)$ & $66.3(58.0-75.8)$ & $53.0(45.5-61.7)$ \\
\hline
\end{tabular}

MLNS, Mediastinal lymph node sampling; $M L N D$, mediastinal lymph node dissection; $C I$, confidence interval.

observe a difference in the recurrence rates or pattern of recurrence between MLND and MLNS groups. Previous reports from this trial showed no increase in morbidity or mortality with the addition of MLND. ${ }^{6}$

After Evarts A. Graham's first successful pneumonectomy, surgical resection for lung cancer became popular in the United States. Pneumonectomy remained the mainstay of therapy until it was challenged by Johnson and colleagues. ${ }^{8}$ Subsequently, in a 1962 report by Shimkin and colleagues, ${ }^{9}$ lobectomy was shown to be acceptable therapy. Although the extent of resection was reduced, removal of all mediastinal lymph tissue was still considered necessary, extrapolating from other solid tumors, such as breast or gastric cancer, for which removal of all the draining lymph nodes was considered the standard of care. In a report on complete mediastinal lymphadenectomy in 1951, Cahan and colleagues ${ }^{10}$ (from the Memorial Sloan Kettering Cancer Center) found "that some patients experienced longterm survival when the positive regional lymph nodes also were removed." In a subsequent review, Martini ${ }^{11}$ commented that complete MLND led to "more favorable long-term survival." Thus, complete MLND has been considered the standard of care for lung cancer resection at most academic centers.

This "standard" has not been followed by the majority of surgeons. In a review of surgical care in the United States in 2001, Little and colleagues ${ }^{2}$ found that only $57.8 \%$ of patients who had surgery as their initial treatment of lung cancer had any mediastinal lymph nodes sampled or removed. In community hospitals, the rate of any surgical lymph node assessment was even lower at only $48.1 \%$.

MLND improves staging accuracy by increasing lymph node harvest and improving identification of occult N2 disease. ${ }^{12-14}$ However, whether survival is improved by MLND is controversial. In a subgroup analysis of patients with stage II or IIIA NSCLC entered into Intergroup trial 0115 of adjuvant chemoradiotherapy versus radiotherapy after resection, Keller and colleagues ${ }^{15}$ reported improved longterm survival in patients with right upper lobe tumors who had MLND with a median survival of 57.5 months versus MLNS with a median survival of 29.2 months. The choice of MLND versus MLNS was not randomized and was based on surgeon preference. In contrast with the Z0030 study,
Keller and colleagues' analysis included only patients who had positive N1 or N2 nodes. There was no difference in the recurrence rates between the 2 cohorts.

A randomized trial comparing MLND and MLNS in 169 patients with stage I, II, or IIIA NSCLC reported by Izbicki and colleagues ${ }^{16}$ found no significant difference in survival after a median follow-up of 47.5 months. In the MLND group, lymph nodes were removed from stations 12, 11, $10,7,4$, and 5 in all patients, whereas in the sampling group mediastinal nodes were removed only if they appeared suspicious. At a median follow-up of 47.5 months, there was no difference in overall or disease-free survival except in patients with $\mathrm{N} 1$ or single-station N2 nodes in whom both overall and disease-free survival was improved. Although their sampling methodology was a bit different than ours, they also found only a small number of patients $(5.5 \%)$ had unsuspected mediastinal lymph node involvement after MLND.

By using a protocol similar to that in Izbicki and colleagues' study ${ }^{16}$ in patients with clinical stage I small $(<2 \mathrm{~cm})$ T1 NSCLC (87\% nonsquamous cancers), Sugi and colleagues ${ }^{14}$ found no difference in survival between the MLND and MLNS groups (5-year survival: $81 \%$ and $84 \%$, respectively). Unsuspected N2 disease was identified in $12 \%$ and $14 \%$ of MLND and MLNS groups, respectively.

However, a larger randomized trial of 532 patients with clinical stage I, II, or IIIA NSCLC reported by Wu and colleagues, ${ }^{3}$ comparing MLND with MLNS, reported significantly improved survival with MLND. The median survival was 43 months in the MLND group and only 32 months in the MLNS group $(P=.0001)$. By multivariate analysis, the number of lymph node metastases, type of nodal dissection (MLND vs MLNS), tumor size, and pathologic stage were all significant prognostic factors.

In contrast with those in the ACOSOG Z0030 trial, the patients in the trial reported by $\mathrm{Wu}$ and colleagues ${ }^{3}$ were only staged clinically before randomization. As a result, $48 \%$ of the patients in the MLND had pathologic stage IIIA disease versus $28 \%$ in the MLNS arm. Furthermore, in the MLNS arm, mediastinal nodes were sampled only if suspicious $(>1 \mathrm{~cm})$ or hard. Unlike in the ACOSOG Z0030 trial, systematic sampling of the mediastinal nodes was not performed. 
A meta-analysis of these 3 trials reported a survival advantage for stages I, II, and IIIA, and a fixed-effects model reported by the same group reported MLND reduced the risk of death for early-stage disease. ${ }^{17,18}$

The ACOSOG Z0030 protocol required systematic sampling of mediastinal nodes by mediastinoscopy, thoracotomy, or VATS. In addition, any suspicious nodes were also sampled. Only after all required node stations were proven to be negative for metastatic disease was the patient eligible for randomization to no further lymph node removal (MLNS) or to formal complete lymph node dissection (MLND). This process allowed us to eliminate many patients who may have had "occult" positive mediastinal lymph nodes and explains the low (4\%) incidence of unsuspected N2 disease found in patents who underwent complete mediastinal lymphadenectomy. In this regard, the Z0030 trial differs from previous randomized trials in that all patients truly had early-stage disease.

Lymph node sampling by mediastinoscopy, VATS, or thoracotomy was equally efficacious in accomplishing systematic lymph node sampling. Endobronchial ultrasound-transbronchial needle aspiration and endoscopic ultrasound-fine-needle aspiration were not evaluated in this trial.

Clinical staging with computed tomography (CT) and positron emission tomography is not equivalent to surgical staging; thus, in the absence of surgical staging of the mediastinal lymph nodes as was performed in the Z0030 trial, MLND is essential for both accurate staging and improved survival, as suggested by $\mathrm{Wu}$ and colleagues. ${ }^{3}$ In patients who are surgically staged as in our trial, MLND identifies truly occult $\mathrm{N} 2$ disease and thereby provides such patients with the opportunity to receive adjuvant chemotherapy, which has now been shown to improve survival. At the time this study was performed, adjuvant chemotherapy was not the standard of care, which may have contributed to the lack of survival benefit in the MLND arm.

Determination of the amount of lymph node tissue removed in both arms of the trial was based on the number or fragments of nodes rather than the weight, and this has some inherent inaccuracies. This is a limitation of the study and may have contributed to the lack of difference between the 2 arms of the trial.

This study was conducted by many surgeons, all American Board of Thoracic Surgery (or equivalent) certified and in many institutions (both community and academic); thus, the results are generalizable and not limited to a few specialized surgeons or centers. The breadth of surgical participation also introduced a potential problem of standardization. However, because each operative and pathology note was reviewed by one of the principle investigators (M.S.A. or G.E.D.), the variation was minimized, and patients with inadequate MLND or overly aggressive sampling were deemed ineligible.

\section{CONCLUSIONS}

MLND does not improve long-term survival in patients with early-stage (T1 or T2, N0 or nonhilar N1) NSCLC who have pathologically negative mediastinal and hilar nodes after rigorous systematic preresection lymph node sampling. In such patients, MLND also does not affect the rate of local or regional recurrence. Our results do not apply to patients with $\mathrm{T} 3$ or T4 tumors or those with known hilar or N2 disease because they were not included in our study. Staging by positron emission tomography-CT or CT alone is not equivalent to the invasive staging performed in this study, and surgeons cannot use this study to justify excluding invasive mediastinal staging from their evaluation of patients with early-stage NSCLC.

MLND provides patients with the most accurate staging and the opportunity for adjuvant therapy if occult metastatic disease is present. Because current preoperative staging cannot definitively identify patients with mediastinal lymph node involvement, and because patients with known hilar or mediastinal disease (N2) or with T3 or T4 tumors may benefit from MLND because the pretest probability of N2 disease is higher, we still recommend that all patients with resectable NSCLC undergo MLND because the procedure does not increase mortality or morbidity.

The authors thank the late Dr Robert J. Ginsberg for valuable leadership in designing this trial.

American College of Surgeons Oncology Group Z0030 TrialAbramson Cancer Center of the University of Pennsylvania; Shrager, Joseph B.; Allegheny Cancer Center Network, Keenan, Robert J., Landreneau, Rodney J.; Beth Israel Deaconess Medical Center, Boston, MA, LoCicero, Joseph, Thurer, Robert Lee; Beth Israel Medical Center, New York, NY, Keller, Steven M.; Bethesda North Hospital, Buckley, Donald C.; Cedars-Sinai Medical Center, McKenna, Robert J.; Central Baptist Hospital; Creighton University Medical Center, Scott, Walter J. Veterans Affairs Medical Center, Mitchell, John D.; Edward Hospital, Bleck, Phyllis C.; Englewood Hospital and Medical Center: Fox Chase Cancer Center, Goldberg, Melvyn, Scott, Walter J.; Good Samaritan Hospital (Cincinnati), Smith, J. Michael; Holmes Regional Medical Center, Greene, Michael A., Malias, Mark A.; Huntington Memorial Hospital, Cohen, Robbin G., Jameson Hospital, Landreneau, Rodney J.; Jewish Hospital, Bowling, Roy G.; Lankenau Medical Research Center, Carp, Ned Z.; Latter Day Saints Hospital, Collins, Michael P.; Lenoir Memorial Hospital, Whitlark, Joseph D.; London Health Sciences Centre (University of Western Ontario Medical Center), Inculet, Richard I., Malthaner, Richard A.; Loyola University Medical Center, Vigneswaran, Wickii T.; MD Anderson Cancer Center (University of Texas), Putnam, Joe B., Rice, David Christopher, Roth, Jack A., Vaporciyan, Ara A.; Mary Imogene Bassett Hospital, Ryan, M. Bernadette; Massachusetts General Hospital, Gaissert, Henning A.; Mayo Clinic (Rochester), Allen, Mark S., Deschamps, Claude, Miller, Daniel L., Nichols, Francis C.; Medical Center of Central Georgia; Medical Center of Southwest Louisiana, Lirtzman, Mitchell D.; Medical College of Virginia Hospital (Virginia Commonwealth University), Cohen, Neri M.; 
Memorial Medical Center (Southern Illinois University School of Medicine), Hazelrigg, Stephen R.; Memorial Sloan-Kettering Cancer Center, Bains, Manjit S., Rusch, Valerie W. Robert J. Downey, Robert J. Ginsberg; Mobile Infirmary Medical Center, Bradley Scott, Walker, Gaylord T.; New York Hospital-Cornell University Medical Center, Altorki, Nasser K., Port, Jeffrey L.; Omaha Veterans Administration Medical Center, Scott, Walter J.; Peter MacCallum Cancer Institute, Wright, Gavin M.; Providence Portland Medical Center, Douville, Emery Charles, Handy, John R., Ott, Gary Yee, Tsen, Andrew C.; Rhode Island Hospital, Gaissert, Henning A.; Roswell Park Cancer Institute, Anderson, Timothy M., Demmy, Todd L., Nwogu, Chukwumere E.; Saint Clair Hospital, Landreneau, Rodney J., Saint John's Hospital, Hazelrigg, Stephen R.; Saint Luke's Hospital of Duluth, Streitz, John M.; Saint Thomas Hospital (Nashville), Nesbitt, Jonathan C.; Saint Vincent Hospital (Green Bay), Coleman, Edward J.; Saint Vincent's Hospital, Melbourne, Wright, Gavin M; Stanford University (Hospital), Whyte, Richard I.; Stony Brook University Hospital; Thomas Jefferson University Hospital, Pechet, Taine T.; Toronto General Hospital, Darling, Gail E., Johnston, Michael R., Keshavjee, Shafique, Pierre, Andrew F., Waddell, Thomas K.; Trinity Cancer Care Center (Minot), Rothberg, Martin L.; University of California San Francisco Medical Center (Long-Moffitt), Jablons, David M.; University of California, Irvine, Milliken, Jeffrey C.; University of Chicago (Hospitals), Ferguson, Mark K.; University of Cincinnati Medical Center, Howington, John A., Reed, Michael F.; University of Miami, Katariya, Kushagra, Thurer, Richard J.; University of Missouri-Ellis Fischel, Demmy, Todd L.; University of Pittsburgh-Presbyterian Hospital, Buenaventura, Percival O., Keenan, Robert J., Landreneau, Rodney J., Luketich, James D.; University of Pittsburgh (Shadyside Hospital), Christie, Neil A., Landreneau, Rodney J., Luketich, James D.; University of Pittsburgh Medical Center-St Margaret Hospital, Landreneau, Rodney J.; University of Rochester (Medical Center), Feins, Richard H., Johnstone, David W., Watson, Thomas J.; University of South Alabama, LoCicero, Joseph; University of Virginia (Medical Center), Daniel, Thomas M., Jones, David R.; University of Washington Medical Center, Vallieres, Eric, Wood, Douglas E.; University of Wisconsin, Weigel, Tracey L.; Upstate Medical University (SUNY-Upstate Syracuse), Dexter, Elizabeth U., Kohman, Leslie J.; VA Medical Center - Cincinnati, Howington, John A., Reed, Michael F.; Valley Hospital, Lee, Youngick, Tsoukas, Elias N.; Veterans Administration Center, Seattle (Puget Sound), Vallieres, Eric; Veterans Administration Medical CenterMinneapolis, Kelly, Rosemary F.; Washington University (Barnes Jewish Hospital), Battafarano, Richard J., Cooper, Joel D., Meyers, Bryan F., Patterson, G. Alexander; West Virginia University (Mary Babb Randolph Cancer Center), Graeber, Geoffrey M.; Western Pennsylvania Hospital, Keenan, Robert J., Landreneau, Rodney J.; Westmoreland Hospital, Keenan, Robert J., Landreneau, Rodney J.; William Beaumont Hospital (Royal Oak), Chmielewski, Gary W.

\section{References}

1. Altekruse SF, Kosary CL, Krapcho M, et al, eds. SEER Cancer Statistics Review, 1975-2007. Bethesda, MD: National Cancer Institute. Available at: http://seer. cancer.gov/csr/1975_2007/, based on November 2009 SEER data submission, posted to the SEER website, 2010.

2. Little AG, Rusch VW, Bonner JA, et al. Patterns of surgical care of lung cancer patients. Ann Thorac Surg. 2005;80:2051-6.
3. Wu Y, Huang ZF, Wang SY, et al. A randomized trial of systematic nodal dissection in resectable non-small cell lung cancer. Lung Cancer. 2002;36:1-6.

4. Caglar HB, Baldini EH, Othus M, et al. Outcomes of patients with stage III nonsmall cell lung cancer treated with chemotherapy and radiation with and without surgery. Cancer. 2009; 115:4156-66.

5. Mountain CF, Dresler CM. Regional lymph node classification for lung cancer staging. Chest. 1997;111:1718-23.

6. Allen MS, Darling GE, Pechet TT, et al. Morbidity and mortality of major pulmonary resections in patients with early-stage lung cancer: initial results of the randomized, prospective ACOSOG Z0030 trial. Ann Thorac Surg. 2006;81:1013-20.

7. Darling GE, Allen MS, Decker PA, et al. Number of lymph nodes harvested from a mediastinal lymphadenectomy: results of the randomized, prospective ACOSOG ZOO30 trial. Accepted for publication. Chest. 2010 Sep 9 [Epub ahead of print].

8. Johnson J, Kirby CK, Blackmore WS. Should we insist on radical pneumonectomy as routine procedure in the treatment of carcinoma of the lung? J Thorac Surg. 1958;36:309-15

9. Shimkin MB, Connelly RR, Marcus SC. Pneumonectomy and lobectomy in bronchogenic carcinoma: a comparison of end results of the Overholt and Ochsner clinic. J Thorac Surg. 1962;44:503-19.

10. Cahan WG, Watson WL, Pool JL. Radial pneumonectomy. J Thorac Surg. 1951; 22:449-73.

11. Martini N. Mediastinal lymph node dissection for lung cancer. The Memorial experience. Chest Surg Clin N Am. 1995;5:189-203.

12. Doddali C, Aragon A, Barlesi F, Chetaille B, Robitail S. Does the extent of lymph node dissection influence outcome in patients with stage I non-small cell lung cancer? Eur J Cardiothorac Surg. 2005;27:680-5.

13. Gajra A, Newman N, Gamble GP, et al. Effect of number of lymph nodes sampled on outcome in patients with stage I non-small-cell lung cancer. J Clin Oncol. 2003;21:1029-34.

14. Sugi K, Nawata K, Fujita N, et al. Systematic lymph node dissection for clinically diagnosed peripheral non-small-cell lung cancer less than $2 \mathrm{~cm}$ in diameter. World J Surg. 1998;22:290-5.

15. Keller SM, Adak S, Wagner H, et al. Mediastinal lymph node dissection improves survival in patients with stages II and IIIa non-small cell lung cancer. Eastern Cooperative Oncology Group. Ann Thorac Surg. 2000;70:358-65.

16. Izbicki JR, Passlick B, Pantel K, et al. Effectiveness of radical systematic mediastinal lymphadenectomy in patients with resectable non-small cell lung cancer: results of a prospective randomized trial. Ann Surg. 1998;227:138-44.

17. Wright G, Manser RL, Byrnes G, et al. Surgery for non-small cell lung cancer: systematic review and meta-analysis of randomised controlled trials. Thorax. 2006;61:597-603.

18. Manser R, Wright G, Hart D, et al. Surgery for early stage non-small cell lung cancer (Cochrane Review). In: The Cochrane Library, Issue 1. Chichester, UK: John Wiley \& Sons; 2005.

\section{Discussion}

Dr Joseph Shrager (Stanford, Calif). I have no conflicts. I congratulate Dr Darling and coauthors for a carefully conducted study on a subject that has daily practical relevance. We heard from this data set in 2005 that lymph node dissection causes no increased morbidity or mortality versus sampling, and today's presentation gives us the long-awaited survival data. The results, as you heard, show that there is no difference in either overall or disease-free survival whether one does a complete dissection versus an aggressive sampling in the early stages that were studied. But despite these findings, you recommend that a complete dissection is the procedure of choice, because, and I quote loosely, "it improves the accuracy of staging and thus the selection of patients for adjuvant therapy."

I would agree with your conclusion that complete dissection remains preferable, but for completely different reasons than those you describe. Only $4 \%$ of additional patients were found to have positive nodes at dissection that were missed at sampling, and presumably most of these patients then received adjuvant chemotherapy, yet there was still no survival advantage to the dissection. 
So I don't understand why "better staging" is a good reason to do a complete dissection, and I don't think that surgeons will buy that reasoning, honestly.

My reasoning for continuing to do complete dissections would be that the sampling stipulated in the protocol is not at all a realworld sampling, and few surgeons would actually be doing that type of extensive sampling. With a less extensive, more real-world sampling, you would have found more than $4 \%$ additional nodes at complete dissection, and so you would possibly also have found a significantly increased survival. So I think it is dangerous for everyone to leave the meeting thinking that sampling is okay. As you emphasized, sampling is only okay if you have done this particular systematic, extensive sampling with frozen sections as stipulated in the study, and that is a sampling that pretty much approached being a total lymphadenectomy. If your sampling is going to be that extensive, it is probably more efficient to just do a lymphadenectomy from the start. I have 3 specific questions.

First, the article describes a power analysis that arrived at a target accrual of 1037, which you nearly achieved, but I would guess that that power analysis was based on a likelihood of identifying more than only $4 \%$ additional nodes at the time of the lymphadenectomy. So are you concerned that you have sort of a type II error, that is, a false-negative result?

Dr Darling. First, my comment on your comment, and that is we know from the previous pattern of care study that you are correct. In fact, less than half the patients who have surgery for NSCLC in North America actually have any kind of lymph node sampling performed at mediastinoscopy or before resection. So these results don't reflect the real world but may inform real-world practice, and I would echo your comments that the patients were rigorously staged and our results don't apply to patients who don't have that kind of sampling. This is supported by the literature. The survival benefit for lymph node dissection shown in studies was in a group of patients who did not have any kind of preresection sampling or staging, and therefore their finding of so-called occult N2 disease was much higher.

In terms of your question about the power, the overall sample size was based on the primary aim of comparing overall survival between MLND and MLNS. Because of the limited number of subjects enrolled with higher stage disease (ie, $\mathrm{pN} 1$ and pN2/pIIIA), the power for the study was amended. This amendment was approved by the National Cancer Institute. The amended power calculation presented in the article assumes the following proportions for stage groups pT1N0 (41\%), pT2N0 (41\%), pN1 (13\%), and $\mathrm{pN} 2 / \mathrm{IIIA}(4 \%)$ in the MLNS arm. The power calculation was based on a 1 -sided, alpha $=0.05 \log$ rank test for $90 \%$ power, which resulted in needing to enroll 1037 subjects. We enrolled 1111 subjects, of whom 1023 were deemed eligible. As described in the article, both intent-to-treat analyses on all randomized subjects and analyses limited to the eligible subjects were performed. Various power scenarios were considered for the secondary aim of identifying occult metastases. For example, if $5 \% \mathrm{pN} 2$ nodes were discovered in the MLNS versus $10 \%$ in the MLND dissection arm, there would be more than $90 \%$ power. On the basis of the observed differences, we think the study was adequately powered.

Dr Shrager. You showed us the results in the T1 and T2 subgroups. I wonder about the N0 and N1 subgroups. Because there is certainly going to be more occult N2 disease in those with clinical N1 than in those with clinical N0, most of us would be pretty concerned about leaving out a complete lymphadenectomy in somebody who has clinical N1.

Dr Darling. The study protocol mandated that only patients with nonhilar N1 were eligible and the hilar nodes had to be sampled, proven to be negative by frozen-section for the patient to be eligible. The actual number of patients with N1 is relatively small. So any N1 nodes that were present were actually within the lobectomy specimen. This is similar to the real world: The surgeon would not know the status of the intralobar nodes, only the hilar nodes. So, in the study population, the highest resected nodes were negative.

Dr Shrager. I wonder if you think this study has any relevance to the role of VATS lobectomy? As you know, many continue to find that doing a true complete mediastinal lymphadenectomy is difficult to do thoracoscopically. However, if complete dissection is of little benefit over a good sampling, is the last barrier to a wider acceptance of VATS lobectomy for earlier stage disease gone?

Dr Darling. If we are going to do lung cancer surgery by VATS, we need to do the same operation that we do when we do it open, and that includes a lymph node dissection or a rigorous sampling, and I don't think it is appropriate for us to just simply go in and take out the lobe and not do a proper sampling of the lymph nodes. Only $7 \%$ of the patients in this study were resected by VATS, and that was primarily done by one surgeon. Dr Allen and I reviewed all the path and operative notes from all the patients in the trial, and particularly we reviewed those VATS cases because of our bias that maybe they wouldn't have had an adequate dissection or sampling. In that particular surgeon's hands, an adequate dissection was done and the lymph node counts were comparable to those taken with open surgery. So I think it is possible to do a good lymph node dissection or sampling with VATS technique, and it behooves us as thoracic surgeons who treat lung cancer to do the same job with VATS as we do with open.

Dr Thomas D'Amico (Durham, NC). Could you just clarify how you did the audit to make sure that a sampling or a dissection was done?

Dr Darling. As I mentioned, we reviewed all the operative notes and looked at the numbers of lymph nodes that were actually removed, and we actually disqualified some patients because they had excessive sampling or had not had an adequate dissection. Dr Allen and I both reviewed those results. We eliminated some of the patients because they didn't follow the protocol.

Dr D'Amico. What percentage of patients were upstaged so that treatment was changed? Were any of the $4 \%$ of $\mathrm{N} 2$ also N1 so that they would have received chemotherapy anyway?

Dr Darling. Of the 21 patients, 11 had N1 disease. This trial was completed in 2004. So at that time, adjuvant chemotherapy was not recommended for N1 disease. In reference to Dr Shrager's previous comment, these patients did not necessarily receive adjuvant therapy at that time, but nowadays they would, and this may have improved survival.

Dr Marcin Zielinski (Zakopane, Poland). I have 2 questions. First, what was the mean number of nodes removed during lymphadenectomy in the lymphadenectomy group?

Dr Darling. The median number of N2 nodes taken was 11 on the right and 12 on the left, but if all nodal stations are included, the median number of nodes taken was 18 . Ninety-nine percent of our patients had 6 or more nodes taken from 3 mediastinal lymph node 
stations and $90 \%$ had 10 or more nodes removed from 3 mediastinal lymph node stations.

Dr Zielinski. My second question is, do you know how many patients were positive on the initial sampling with frozen-section?

Dr Darling. We don't have that data because they were not eligible to be randomized.

Dr Paul De Leyn (Leuven, Belgium). That was a nice presentation. When you read the article in 2005, which describes morbidity and mortality, you indeed think that the sampling is already extensive, and this is important. Can you describe from a technical point of view when you sample, let's say, station 7 , is this just picking out a few nodes or is this removing this whole station? What is the difference between sampling and dissection in your study?
Dr Darling. The sampling protocol specified that you were simply directed to sample one node from each station. If there were any suspicious-looking nodes, obviously they were also required to be sampled as opposed to doing a formal clean-out of the area. So the protocol did specify to sample 1 node. Occasionally the surgeons would take more than 1 node, but, as I mentioned, if they took a whole lot of nodes (eg, 5-7 nodes from station 7), we disqualified those as being a dissection rather than a sampling. For the lymph node dissection, the protocol specified removing all lymph node-bearing tissues between anatomic boundaries. For example, for stations $2 \mathrm{R}$ and $4 \mathrm{R}$ all tissue between the superior vena cava, trachea, right innominate artery, and the right bronchus was removed, not just the visible lymph nodes.

\section{COMMENTARY}

\section{Less is more... (more or less...)}

\section{Sudish C. Murthy, MD, PhD}

In this month's issue, Dr Darling and colleagues present the results of a long-awaited multicenter, prospective, randomized trial comparing efficacy of mediastinal lymph node sampling (MLNS) and mediastinal lymph node dissection (MLND) for localized non-small cell lung cancer (NSCLC). This study was one of the many "brain-children" of the late Dr Robert J. Ginsberg, and its organization, attention to detail, and insight speak to the lasting legacy of this pioneer. In addition, the group of distinguished authors who carried this trial to its conclusion has overcome enormous obstacles to complete this ambitious enterprise, dutifully and meticulously organizing data collected from over 100 surgeons at 63 member institutions while maintaining some element of quality control throughout the process - truly a formidable task whose complexity should be appreciated.

The importance of this trial cannot be underestimated inasmuch as the role of lymphadenectomy for NSCLC, until now, has served as a point of confusion for most thoracic

From the Cleveland Clinic Foundation, Department of Thoracic and Cardiovascular Surgery, Cleveland, Ohio.

Disclosures: Authors have nothing to disclose with regard to commercial support.

Received for publication Jan 13, 2011; revisions received Jan 13, 2011; accepted for publication Jan 13, 2011.

Address for reprints: Sudish C. Murthy, MD, PhD, Cleveland Clinic Foundation/Desk J4-1, Department of Thoracic and Cardiovascular Surgery, 9500 Euclid Ave, Cleveland, OH 44195 (E-mail: murthys1@ccf.org).

J Thorac Cardiovasc Surg 2011;141:670-72

$0022-5223 / \$ 36.00$

Copyright @ 2011 Published by Elsevier Inc. on behalf of The American Association for Thoracic Surgery

doi:10.1016/j.jtcvs.2011.01.024 surgical oncologists. Although widely heralded as an important adjunct for management of the disease, there has been little evidence to support this grandiose contention in clinical practice. In fact, a shockingly high number of lung cancer operations are performed without a single mediastinal lymph node even being sampled. ${ }^{1}$ Yet, despite this, sophisticated and detailed guidelines of how MLND should be integrated into NSCLC management have continued to emerge. ${ }^{2}$ At last, there is a well-done, randomized trial to support a position on the subject.

The findings of this trial are provocative, as surprisingly, equivalence was found between MLNS and MLND for patients with early NSCLC (N0 or nonhilar N1 cancers). This, in part, corroborates results from a similar, albeit much smaller and less well-controlled, trial from a decade ago. ${ }^{3}$ But the results appear to fly against the popular notion that MLND is a superior surgical technique with important survival benefits for patients with early-stage NSCLC. ${ }^{4-7}$ None of these lesser studies was randomized or as well conceived, however. Importantly, after careful review of each study, including my own, unfortunately, the authors of the latter group of manuscripts need not ready their retraction statements just yet, inasmuch as there may still be room for peaceful coexistence between studies, because slightly different questions were addressed by each.

There is a reason why such randomized studies are completed so infrequently and why this trial casts such a heroic specter. Clearly, it is difficult to have equipoise for such a trial if one believes strongly (either way) about MLND, 